\title{
Avaliação do seguimento de lesões precursoras de câncer do colo do útero - uma revisão bibliográfica
}

\author{
Gabriela Henrique Oliveira Cavalcante ${ }^{1}$ (D) \& Gilson Jácome dos Reis ${ }^{1,2,3}$
}

(1) Fundação Oswaldo Cruz, Escola Nacional de Saúde Pública, Curso de Especialização em Gestão de Redes de Atenção à Saúde, Rua Leopoldo Bulhões 1480, Manguinhos 21041-210, Rio de Janeiro, Brasil. E-mail: gabycavalcante.18@gmail.com

(2) Universidade Federal do Rio de Janeiro, Hospital Universitário Clementino Fraga Filho, Rua Rodolpho Paulo Rocco 255, Cidade Universitária 20911-270, Ilha do Fundão, Rio de Janeiro, Brasil. E-mail: gilson_jacome@yahoo.com.br

(3) Fundação Oswaldo Cruz, Escola Nacional de Saúde Pública, Programa de Pós-Graduação em Saúde Pública, Rua Leopoldo Bulhões 1480, Manguinhos 21041-210, Rio de Janeiro, Brasil.

Cavalcante G.H.O. \& Reis G.J. (2021) Avaliação do seguimento de lesões precursoras de câncer do colo do útero - uma revisão bibliográfica. Pesquisa e Ensino em Ciências Exatas e da Natureza, 5: e1741. http://dx.doi.org/10.29215/pecen.v5i0.1741

Editora acadêmica: Sandra Maria Souza da Silva. Recebido: 10 março 2021. Aceito: 09 julho 2021. Publicado: 25 julho 2021.

Resumo: O câncer do colo do útero, também chamado de câncer cervical, está relacionado com a infecção de alguns tipos do Papilomavírus Humano (HPV) chamados oncogênicos. Este tipo de tumor é o quarto mais frequente na população feminina, com aproximadamente 570 mil novos casos por ano no mundo. Cerca de $7.5 \%$ da população feminina é acometida por este câncer e 311 mil mulheres evoluem para óbito. $\mathrm{O}$ exame preventivo (Papanicolau) é uma medida fundamental para detecção precoce de lesões precursoras e amplamente utilizado. Quando a lesão é diagnosticada precocemente o tratamento apresenta alto grau de cura. $\mathrm{O}$ rastreamento e acompanhamento destes casos são essenciais para evitar o surgimento de novos casos de câncer que implicam em tratamentos mais complexos e dispendiosos. Este estudo consiste em uma revisão bibliográfica referente a avaliação e condução do seguimento de lesões precursoras deste tipo de câncer. Os dados analisados evidenciam dificuldades encontradas pelas mulheres em agendar consultas de retorno após realização do exame preventivo, o que dificulta a implementação de ações imediatas para o tratamento. A perda de seguimento é um problema relevante e ocorre devido a fatores como a má interpretação de exames, resultando em repetição desnecessária do exame preventivo e atraso na realização de colposcopia e, consequentemente, do início de tratamento; ausência de seguimento em exames alterados; deficiência de orientação e encaminhamento oportuno destas mulheres. Para enfrentar tais desafios propõe-se que os serviços estejam organizados em rede, que inclui sistema de rastreamento conectado com o seguimento e tratamento adequado das mulheres que apresentem alterações nos exames preventivos. Também se faz necessário investigação sobre os motivos de não adesão das mulheres, controle de qualidade dos serviços de coleta e análise das amostras, avaliação de protocolos utilizados e busca ativa das pacientes para realização do exame Papanicolau.

Palavras-chave: Atenção à Saúde, exame Papanicolau, longitudinalidade, detecção precoce de câncer.

Evaluation of the follow-up of precursing cervix uteri cancer injuries - a bibliographic review

Abstract: Cervical cancer, also called cervical cancer, is related to the infection of some types of Human Papillomavirus (HPV) called oncogenic. This type of tumor is the fourth most frequent in the female population, with approximately 570 thousand new cases per year worldwide. About $7.5 \%$ of the female population is affected by this cancer and 311.000 women evolve to death. The preventive exam (Pap) is a fundamental measure for early detection of precursor lesions and is widely used. When the lesion is diagnosed early, the treatment presents a high degree of cure. Tracking and monitoring these cases are essential to avoid the emergence of new cases of cancer that require more complex and expensive 
treatments. This study consists of a literature review regarding the assessment and follow-up of precursor lesions of this type of cancer. The data analyzed show difficulties encountered by women in scheduling return appointments after the preventive examination, which makes it difficult to implement immediate actions for treatment. Loss to follow-up is a relevant problem and occurs due to factors such as misinterpretation of exams, resulting in unnecessary repetition of the preventive exam and delay in performing colposcopy, and consequently in the initiation of treatment; lack of follow-up in altered exams; lack of timely guidance and referral of these women. To face such challenges, it is proposed that the services be organized in a network, which includes a tracking system connected with the adequate followup and treatment of women who present alterations in preventive exams. It is also necessary to investigate the reasons for women's non-adherence, quality control of sample collection and analysis services, evaluation of the protocols used and active search for patients to undergo Pap smears.

Key words: Health care, Papanicolaou test, longitudinality, early detection of cancer.

\section{Introdução}

O câncer do colo do útero, também chamado de câncer cervical, é causado pela infecção persistente por alguns tipos do Papilomavírus Humano (HPV) chamados oncogênicos. Pelo menos 13 tipos de HPV são considerados oncogênicos, apresentando maior probabilidade de provocar infecções, especialmente os tipos HPV-16 e HPV-18, responsáveis por cerca de $70 \%$ dos cânceres cervicais. A infecção genital por este vírus é muito frequente e não causa doença na maioria das vezes, regredindo espontaneamente. Além da infecção por HPV, o início precoce da vida sexual, a multiplicidade de parceiros sexuais e o tabagismo são fatores de risco para o desenvolvimento do câncer do colo do útero. $\mathrm{O}$ exame citológico de Papanicolau, também conhecido como exame preventivo é o método de rastreamento recomendando pelo Ministério da Saúde no Brasil para rastreamento de lesões precursoras do câncer do colo do útero em mulheres de 25 a 64 anos e que já tiveram atividade sexual. Essa faixa etária foi priorizada pelo fato de ser a maior com ocorrência das lesões de alto grau e serem passíveis de tratamento eficazes para não evoluir para o câncer. Além do rastreamento, as medidas de prevenção e controle do câncer de colo de útero incluem a vacinação contra o HPV e ações educativas (Febrasgo 2017; Brasil 2021a).

A vacina HPV foi incluída ao calendário nacional do Programa Nacional de Imunização (PNI) no ano de 2014. Trata-se de uma vacina tetravalente contra os tipos 6, 11, 16 e 18 do HPV (Santos \& Dias 2018). É recomendado pelo Ministério da Saúde a aplicação de duas doses para meninas entre 9 e 14 anos e meninos de 11 a 14 anos. Os tipos 6 e 11 estão relacionados com o desenvolvimento do condiloma acuminado e os 16 e 18 com o desenvolvimento do câncer cervical (Brasil 2021b).

Medidas educativas voltadas para a promoção da saúde para prevenção do câncer do colo do útero compreendem orientações sobre higiene pessoal, uso de preservativos visando prevenir infecções sexualmente transmissíveis (IST), orientações sobre os riscos do uso do tabaco e incentivo a atividade física (Casarin \& Picolli 2011).

O exame citopatológico passou a ser utilizado, ainda na década de 1940, como uma ferramenta valiosa para o diagnóstico precoce do câncer de colo do útero (CCU), por se tratar de um procedimento com alta precisão, baixo custo e execução simples (Caetano et al. 2006). Este exame é eficiente e muito utilizado para detectar as lesões precursoras do câncer do colo do útero, principalmente em fases iniciais, quando o tratamento apresenta alto índice de cura, diminuindo a mortalidade, tornando baixo o custo efetivo (Tavares et al. 2007).

Este tipo de tumor é o quarto mais frequente na população feminina com aproximadamente 570 mil casos novos por ano e responsável por cerca de 311 mil óbitos. Prova de que o país avançou na sua capacidade de realizar diagnóstico precoce é que na década de 1990, 70\% dos casos diagnosticados eram da doença invasiva, ou seja, o estágio mais agressivo da doença. Atualmente, $44 \%$ dos casos são de lesões precursoras do câncer, classificadas in situ do tipo localizada (Brasil 2021c). 
No Brasil, em 2020, foram registrados 16.710 casos novos de câncer do colo do útero e 6.596 dessas mulheres evoluíram para óbito. Particularmente na região Nordeste, o câncer do colo uterino figura entre os mais incidentes no sexo feminino e Pernambuco está entre os estados com maior incidência da doença (Brasil 2021d).

Estima-se uma redução em cerca de $80 \%$ da mortalidade por esse câncer através do rastreamento de mulheres na faixa etária de 25 a 64 anos com o diagnóstico precoce e o tratamento das lesões precursoras (Brasil 2010). A rotina recomendada para o rastreamento no Brasil, se dá a partir de dois exames citopatológicos repetidos a cada três anos, após dois exames normais consecutivos realizados em um intervalo de um ano. $\mathrm{O}$ rastreamento no Brasil ocorre de maneira oportunística ou passiva, isto é, depende da procura das mulheres pelos serviços de saúde. As mulheres diagnosticadas com lesões intraepiteliais do colo do útero no rastreamento devem ser encaminhadas à unidade secundária para confirmação diagnóstica e tratamento (Brasil 2016). O sistema responsável pelo acompanhamento das informações relativas ao câncer do colo do útero é o Sistema de Informações do Câncer (SISCAN). Por meio desse sistema é possível realizar o monitoramento das ações relacionadas à detecção precoce, à confirmação diagnóstica e ao início do tratamento de neoplasias malignas, através de informações referentes aos exames realizados nas mulheres e a frequência que ocorrem as lesões pré-cancerígenas e do câncer invasivo (Brasil 2013).

Para que ocorra uma diminuição significativa dos novos casos de câncer do colo do útero, os programas de rastreamento devem ser estruturados, visando a redução da mortalidade e incidência desta doença. Com o rastreamento é possível detectar um alto número de mulheres com a doença pré-invasora e as formas invasoras. Quanto mais precoce a detecção ocorre, maiores são as chances de cura. Entretanto, o sucesso desse programa está condicionado a uma boa cobertura de exames para população de risco, qualidade na coleta e interpretação do material, bem como tratamento e acompanhamentos adequados (Vale et al. 2010).

As condutas existentes para as mulheres após realizarem o exame preventivo dependerá do resultado do mesmo. Esta conduta vai da repetição da coleta e análise citopatológica em 6 meses (para lesões de baixo grau - HPV e NIC I - que regridem espontaneamente em $80 \%$ das vezes), até um tratamento cirúrgico, passando pela possibilidade de resolução por meio de um tratamento clínico (Brasil 2016). O tratamento das lesões precursoras do câncer do colo útero deve ser individual e apropriado para cada caso. Essa individualidade ocorre desde o acompanhamento cuidadoso das pacientes as diversas técnicas existentes, que incluem a biópsia, histerectomia, quimioterapia e radioterapia. As modalidades terapêuticas objetivam as terapias que preservem a função reprodutiva e que minimizem a morbidade. $\mathrm{O}$ método ablativo ainda é o mais aceito, porém existe a necessidade de um seguimento para estas pacientes, devido à possibilidade de recidiva local ou de progressão de lesões residuais (Febrasgo 2017).

De acordo com as Diretrizes Brasileiras para o rastreamento do câncer de colo do útero (Brasil 2016), mulheres que apresentem lesões intraepiteliais de alto grau (HSIL) devem ser encaminhadas à unidade de referência para realização de colposcopia. Na presença de achados anormais maiores, Junção escamocolunar (JEC) visível (ZT - Zona de Transformação - tipos 1 ou 2), lesão restrita ao colo e ausente suspeita de invasão ou doença glandular, deverá ser realizado o "Ver e Tratar", ou seja, a excisão tipo 1 ou 2, de acordo com o tipo da ZT. Em locais em que não esteja garantida a qualidade da citologia ou quando o colposcopista não se sentir seguro quanto à relevância dos achados, a biópsia é aceitável.

O tratamento do câncer do colo do útero, conforme prevê a Política Nacional de Atenção Oncológica, deve ser feito nas Unidades de Assistência de Alta Complexidade em Oncologia (UNACON) e nos Centros de Assistência de Alta Complexidade em Oncologia (CACON), que fazem parte de hospitais de nível terciário, habilitados para realização dos procedimentos de maior complexidade. Este nível de atenção deve estar capacitado para determinar a extensão da neoplasia (estadiamento), tratar, cuidar e assegurar a qualidade da assistência oncológica (Brasil 2011).

A finalidade do seguimento é detectar alterações celulares precocemente, garantindo que as intervenções sejam oferecidas oportunamente garantindo o seguimento e o tratamento 
adequado para as mulheres. $\mathrm{O}$ seguimento deve ser oferecido às mulheres garantindo-as acesso aos serviços de saúde, de forma integral, em toda trajetória assistencial (Brasil 2016).

Portanto, o objetivo deste estudo foi avaliar se o seguimento das lesões precursoras do câncer do colo do útero segue adequadamente as recomendações do Ministério da Saúde.

\section{Material e Método}

Trata-se de uma pesquisa de caráter descritivo. De acordo com Gil (2011), o estudo descritivo tem como propósito descrever as peculiaridades de uma determinada população ou fenômenos, podendo também estabelecer ligações entre variáveis e formulação de hipóteses explicativas. Para Köche (2011) o estudo descritivo estuda as relações entre duas variáveis de um dado fenômeno sem manipulá-las.

Em relação à forma de abordagem, esta pesquisa se caracteriza como qualitativa. $\mathrm{Na}$ abordagem qualitativa foca-se um modelo no qual a realidade é consolidada levando em consideração a concepção particular dos sujeitos participantes, vendo-os de forma integral, sendo assim o objetivo é compreender e identificar significados tendo como instrumento o entendimento indutivo e interpretativo que se atribui aos dados descobertos, associados ao problema de pesquisa (Soares 2019).

Quanto aos fins, esta pesquisa qualitativa é do tipo explicativa. Essa modalidade de investigação tem como principal objetivo tornar algo inteligível e justificar os motivos. Visa, portanto, esclarecer quais fatores contribuem, de alguma forma, para a ocorrência de determinado fenômeno. Quanto aos procedimentos técnicos, é do tipo bibliográfica, pois é elaborada a partir de material já publicado, constituído principalmente de livros e artigos publicados em periódicos (Gil 2011).

Trata-se de uma revisão bibliográfica de publicações referentes ao tema. Segundo Vieira \& \& Hossene (2007), as revisões da literatura permitem identificar conhecimentos já sedimentados sobre determinado assunto. Mostra a evolução de conhecimentos discutindo falhas e acertos, com críticas e elogios, destacando o que é realmente interessante. Para a prática constituída em evidências, a revisão bibliográfica é um importante recurso que consiste em uma forma de síntese dos resultados de pesquisas relacionados com um problema específico (Gil 2011). Pode-se dizer que a revisão narrativa consiste em uma avaliação não sistematizada, de algumas publicações sobre o tema escolhido, esta pode incluir livros, artigos, dissertações, teses.

Para a busca das publicações foram utilizados os seguintes descritores em saúde (DesCS) através do operador booleano "AND": câncer do colo do útero; exame Papanicolau; e detecção precoce de câncer. Tal estratégia de busca teve a finalidade de selecionar os estudos, de maneira mais geral, que tratam sobre a temática das medidas de controle do câncer de colo de útero. Posteriormente, houve a seleção das publicações acerca dos problemas relativos ao seguimento das mulheres com alterações nos exames realizados durante o rastreamento.

O levantamento dos artigos no banco de dados da Biblioteca Virtual em Saúde (BVS) ocorreu sem levar em consideração um recorte temporal considerando a busca pelos seus descritores. Delimitou-se como critério de inclusão estudos que contemplavam a temática câncer do colo do útero e avaliação do seguimento. Como critério de exclusão, definiu-se os artigos de língua estrangeira, já que o enfoque do estudo é da realidade brasileira; aqueles cujo título não abrangia o assunto escolhido e/ou não estavam disponíveis na íntegra para consulta gratuita. A partir desta busca foram encontrados 36 artigos e destes foram utilizados 10. Os artigos selecionados foram lidos na íntegra e posteriormente realizada uma análise dos resultados.

\section{Resultados e Discussão}

Há evidências de que o rastreamento de mulheres para lesões precursoras do câncer do colo do útero, por meio do exame de Papanicolau, associada ao tratamento em estágios iniciais, tem resultado em uma redução das taxas de incidência e mortalidade de câncer cervical invasor. Assim, o acompanhamento regular, realizado pelas equipes municipais e estaduais, das 
mulheres com exames alterados, em especial aquelas com diagnóstico de lesão de alto grau (NIC II e NIC III), é imprescindível, pois, estas mulheres, se tratadas adequadamente, não evoluirão para o câncer invasivo, o que contribuirá para a diminuição da incidência do câncer do colo do útero. Mas para o alcance dessa redução, a Organização Mundial da Saúde (OMS) recomenda cobertura de, no mínimo, $80 \%$ da população alvo (25 a 64 anos) e padrão de qualidade do exame, tornando-se os parâmetros mais importantes no âmbito da Atenção Primária em Saúde (APS) para a redução da incidência e da mortalidade pelo câncer de colo do útero (Uchimura et al. 2009).

A informação sobre o tratamento, quando inserido no SISCAN, permite ao gestor municipal e estadual avaliar e acompanhar as ações de controle do câncer do colo do útero em sua área de abrangência, contribuindo para a tomada de decisões.

É na Atenção Básica $(\mathrm{AB})$ que as ações preventivas de educação em saúde, detecção precoce e encaminhamento para tratamento em níveis de maior complexidade devem ocorrer. Mesmo após a cura, o acompanhamento da usuária ainda permanece sob responsabilidade da APS para que se previna recidivas (Uchimura et al. 2009).

De acordo com a análise dos artigos selecionados (Tabela 1) foi identificado que a rede física possui a atenção primária como porta de entrada à assistência à saúde da mulher, seguida pela média e alta complexidade (Farias \& Barbieri 2016). A atenção primária apresenta deficiências de agendamento das mulheres, superlotação e escassez de médicos o que resulta na recusa das mulheres desse nível de atenção na busca por cuidados. Sobre a referência da média complexidade, os resultados mostram falta de equipamentos, insumos e profissionais para $o$ atendimento da demanda (Carvalho et al. 2018).

A perda de seguimento ocorre em mulheres com lesões precursoras, que são orientadas a repetir o exame preventivo desnecessariamente, quando deveriam ser logo encaminhadas para a realização de colposcopia. Outra possibilidade de perda de seguimento refere-se quando as mulheres possuem exames citopatológicos alterados e têm o seguimento ignorado, não sendo orientadas e encaminhadas, conforme as normas preconizadas pelo Ministério da Saúde (Brasil 2016). Segundo Araújo et al. (2014), as condutas equivocadas acarretam atrasos na confirmação do diagnóstico e do tratamento, além de gastos desnecessários na repetição do exame.

Araújo et al. (2014) afirmam que é necessário a redução do número de procedimentos desnecessários para otimizar os recursos disponíveis, porém isso não significa redução do número de coletas do exame citopatológico, mas o desenvolvimento de estratégias para que as mulheres com maior risco de desenvolverem câncer e com pouca frequência de realização de exames tenham acesso ao sistema de saúde.

Dalmolin et al. (2016) ressaltam no estudo que existem mulheres com resultados do exame Papanicolau alterados que necessitam da colposcopia e não apresentam informações em seus prontuários sobre o seguimento dado, sugerindo que essas mulheres não foram submetidas às condutas preconizadas.

Risso \& Soares (2011) encontraram problemas na busca ativa das mulheres devido à inadequação dos registros nos prontuários, dificultando a entrega dos resultados de uma maneira efetiva e que possibilitasse a adoção de intervenções preconizadas.

A situação do trabalho, a dificuldade financeira e de locomoção retardam o tratamento de lesões mais graves. Nos serviços de saúde, destaca-se a dificuldade para agendamento de exames específicos, consulta de retorno, insuficiência de recursos e escassez de profissionais em determinadas regiões do país também foram problemas evidenciados (Carvalho et al. 2018).

Segundo Carvalho et al. (2016), as dificuldades em relação ao agendamento da consulta de retorno podem ser ocasionadas devido à demora do laboratório na entrega dos exames, sobrecarga de trabalho, quantidade insuficiente de profissionais de saúde em cada unidade, problemas em localizar o endereço na realização da busca ativa das mulheres, e elevado número de pacientes com alterações no exame. Um outro problema evidenciado pelo mesmo autor é a desinformação encontrada sobre a realização do exame Papanicolau. 
Tabela 1. Caracterização Bibliométrica e resultados da busca.

\begin{tabular}{|c|c|c|c|}
\hline & Autor & Título & Principais resultados \\
\hline 1 & Franco et al. (2019) & $\begin{array}{l}\text { Seguimento de Resultados Alterados } \\
\text { de Papanicolau nas Unidades Básicas } \\
\text { de Saúde da Cidade de São Paulo. }\end{array}$ & $\begin{array}{l}\text { Além de maior cobertura de exames } \\
\text { preventivos, deve-se agir para dar soluções dos } \\
\text { casos alterados, pois quanto mais exames, maior } \\
\text { probabilidade de mais resultados alterados e } \\
\text { cada vez mais a capacidade de reação está } \\
\text { comprometida engessada. }\end{array}$ \\
\hline 2 & Carvalho et al. (2018) & $\begin{array}{l}\text { Trajetórias assistenciais de mulheres } \\
\text { entre diagnóstico e início de } \\
\text { tratamento do câncer de colo uterino. }\end{array}$ & $\begin{array}{l}\text { A atenção básica apresenta deficiências de } \\
\text { agendamento, superlotação e escassez de } \\
\text { médicos. Média complexidade com falta de } \\
\text { equipamentos, insumos e profissionais para o } \\
\text { atendimento da demanda. }\end{array}$ \\
\hline 3 & Carvalho et al. (2016) & $\begin{array}{l}\text { Acesso ao exame Papanicolau por } \\
\text { usuárias do sistema único de saúde. }\end{array}$ & $\begin{array}{l}\text { Acesso à consulta de retorno, mostrando a } \\
\text { dificuldade das mulheres conseguirem voltar } \\
\text { ao serviço após o exame. }\end{array}$ \\
\hline 4 & Farias \& Barbieri (2016) & $\begin{array}{l}\text { Seguimento do câncer de colo de } \\
\text { útero: Estudo da continuidade da } \\
\text { assistência à paciente em uma região } \\
\text { de saúde. }\end{array}$ & $\begin{array}{l}\text { Rede física possui a atenção primária como } \\
\text { porta de entrada a assistência à saúde da } \\
\text { mulher, seguida pela média e alta } \\
\text { complexidade. }\end{array}$ \\
\hline 5 & Dalmolin et al. (2016) & $\begin{array}{l}\text { Mulheres com } \quad \text { exames } \\
\text { citopatológicos alterados: Avaliação } \\
\text { do seguimento de acordo com as } \\
\text { condutas preconizadas pelo Ministério } \\
\text { da Saúde. }\end{array}$ & $\begin{array}{l}\text { Ressaltam em seu estudo que existem mulheres } \\
\text { com resultados do exame papanicolau alterados } \\
\text { e que necessitam da realização da colposcopia e } \\
\text { não apresentam informações em seus } \\
\text { prontuários sobre o seguimento dado em seu } \\
\text { caso, o que sugere que essas mulheres não } \\
\text { foram submetidas às condutas preconizadas. }\end{array}$ \\
\hline 6 & Araújo et al. (2014) & $\begin{array}{l}\text { Avaliação do Seguimento de } \\
\text { Mulheres com Exames } \\
\text { Citopatológicos Alterados de acordo } \\
\text { com as Condutas Preconizadas pelo } \\
\text { Ministério da Saúde do Brasil em } \\
\text { Goiânia, Goiás. }\end{array}$ & $\begin{array}{l}\text { Mulheres com exames citopatológicos alterados } \\
\text { têm seguimento ignorado e não são orientadas } \\
\text { e encaminhadas conforme as normas } \\
\text { preconizadas pelo MS. }\end{array}$ \\
\hline 7 & Ribeiro et al. (2011) & $\begin{array}{l}\text { Itinerário Terapêutico de Mulheres } \\
\text { com Câncer do Colo do Útero: uma } \\
\text { Abordagem Focada na Prevenção. }\end{array}$ & $\begin{array}{l}\text { Estudo desenvolvido em } \text { Minas Gerais } \\
\text { constatou que a maioria das mulheres não } \\
\text { realiza o citopatológico pela qualidade do } \\
\text { atendimento e organização do trabalho, } \\
\text { relatando a preferência pela agilidade de } \\
\text { serviços privados. }\end{array}$ \\
\hline 8 & Risso \& Soares (2011) & $\begin{array}{l}\text { Avaliação dos registros do exame } \\
\text { citopatológico em uma unidade } \\
\text { básica de saúde em Rio Grande-RS. }\end{array}$ & $\begin{array}{l}\text { Encontrou problemas na busca ativa das } \\
\text { mulheres devido à inadequação dos registros } \\
\text { nos prontuários, dificultando a entrega dos } \\
\text { resultados de uma maneira efetiva e que } \\
\text { possibilitasse adotar as medidas de intervenções } \\
\text { preconizadas rapidamente. }\end{array}$ \\
\hline 9 & Uchimura et al. (2009) & $\begin{array}{l}\text { Qualidade e desempenho das } \\
\text { colpocitologias na prevenção de } \\
\text { câncer de colo uterino. }\end{array}$ & $\begin{array}{l}\text { Para detecção precoce de lesões precursoras é } \\
\text { amplamente utilizado o exame preventivo } \\
\text { (Papanicolau), este é eficiente e quando a lesão } \\
\text { é diagnosticada precocemente o tratamento } \\
\text { apresenta alto grau de cura. O rastreamento e } \\
\text { acompanhamento destes casos é essencial para } \\
\text { evitar o surgimento de novos casos de câncer } \\
\text { que implicam em tratamentos mais complexos } \\
\text { e dispendiosos. }\end{array}$ \\
\hline 10 & Cruz \& Loureiro (2008) & $\begin{array}{l}\text { A Comunicação na Abordagem } \\
\text { Preventiva do Câncer do Colo do } \\
\text { Útero: importância das influências } \\
\text { histórico-culturais e da sexualidade } \\
\text { feminina na adesão às campanhas }\end{array}$ & $\begin{array}{l}\text { Abordagem de comunicação para com as } \\
\text { mulheres almejadas pelos programas de } \\
\text { prevenção ainda se mostra deficiente. } \\
\text { Abordagem efetuada numa linguagem em que } \\
\text { muitas mulheres não se identificam ou se } \\
\text { sentem constrangidas por não terem suas } \\
\text { histórias, vivências e valores contextualizados. }\end{array}$ \\
\hline
\end{tabular}

A demora em retornar ao serviço de saúde e obter a possibilidade de investigação do caso e possível tratamento, faz com que as mulheres desistam de procurar atendimento e deixem de lado a oportunidade de cura.

O Instituto Nacional do Câncer (Brasil 2016), estabelece as diretrizes brasileiras de controle do câncer de colo de útero. Recomenda-se que, após confirmação colposcópica ou 
histológica, o tratamento excisional das lesões intraepiteliais escamosas de alto grau, por meio de exérese da zona de transformação (EZT) por eletrocirurgia. Quando a colposcopia é satisfatória, com achado anormal compatível com a citologia, restrito à ectocérvice ou até o primeiro centímetro do canal endocervical, o procedimento deve ser realizado ambulatorialmente, nas unidades de nível de atendimento secundário, permitindo o tratamento imediato das lesões. O objetivo desta estratégia é facilitar o acesso das mulheres ao tratamento, diminuindo a ansiedade, as possibilidades de perdas no seguimento e os custos da assistência.

O Sistema Único de Saúde (SUS) apresenta fragilidade quanto à integralidade da atenção, uma vez que oferece a realização do citopatológico e falha na consulta de retorno, o que expressa a fragmentação do cuidado. É de extrema importância que a consulta de retorno seja agilizada para evitar o desenvolvimento do câncer de colo do útero. Tais falhas estão relacionadas à problemas de gestão e de subfinanciamento do SUS, que são aspectos não excludentes entre si. A constituição de redes de atenção à saúde é uma função da gestão do SUS, em seus três níveis de governo, sendo uma estratégia relevante para garantir a continuidade e a integralidade da atenção à saúde; e a constituição de redes necessita a provisão regular e suficiente de recursos financeiros para custeio e investimento das ações e serviços de saúde. A Emenda Constitucional 95 que estabelece um teto para as despesas primárias da União inviabiliza a integralidade da atenção à saúde.

De acordo com Silva et al. (2014) há estudos, conduzidos em Recife (PE) e Caruaru (PE), que evidenciam relatos de erros nos diagnósticos iniciais e atrasos nos inícios dos tratamentos, com evoluções das doenças, sendo que, em 90\% dos casos, as mulheres recorrerem a exames particulares devido à escassez de vagas públicas nos centros de tratamento, e também como forma de agilizarem os resultados e iniciarem suas terapias. Não obstante, as mulheres também precisam de conhecimentos pessoais para acessarem aos serviços de referência, recorrendo à ajuda de conhecidos e de parentes, que facilitam os acessos iniciais dessas mulheres aos serviços de média e alta complexidade

Um estudo desenvolvido em Minas Gerais constatou que a maioria das mulheres não realiza o citopatológico pela qualidade do atendimento e organização do trabalho, relatando a preferência pela agilidade de serviços privados (Ribeiro et al. 2011).

Os fatores de risco para o câncer de colo uterino e as medidas efetivas de prevenção e controle são conhecidos. Cruz \& Loureiro (2008) identificam os problemas que contribuem para a não adesão de mulheres aos programas e quais os fatores que dificultam o alcance da cobertura prevista nas campanhas. Apesar do empenho do Ministério da Saúde do Brasil e dos profissionais em alcançar o público pretendido, pode-se considerar que a abordagem de comunicação para com as mulheres almejadas pelos programas de prevenção ainda se mostra deficiente. Esta abordagem pode estar sendo efetuada numa linguagem em que muitas mulheres não se identificam ou se sentem constrangidas por não terem suas histórias, vivências e valores contextualizados.

Para superar esse problema, seria necessária uma nova abordagem de orientação a essas mulheres, fazendo com que elas compreendam a importância da prevenção do câncer do colo do útero (a partir do exame Papanicolau) e o seguimento conforme recomendações preconizadas pelo Ministério da Saúde, bem como aperfeiçoar a operacionalização do serviço. Uma estratégia importante a ser implementada é um atendimento humanizado efetivo, para que as mulheres em situação de risco percebam a importância da realização do exame e superem seus medos e tabus.

A importância do seguimento do câncer do colo do útero se dá pela detecção de complicações, como as endometrites e celulites; detecção de lesões residuais; instituição de tratamento conservador; redução do risco de câncer pós-tratamento conservador e identificação de mulheres com menor necessidade de vigilância (Brasil 2016).

Farias \& Barbieri (2016) apontam falhas na coleta do exame preventivo, o que prejudica a qualidade da amostra e, consequentemente, dos resultados dos exames. A maioria dos profissionais que realizam os exames nunca foram capacitados para atuar na prevenção do câncer do colo do útero; demostram que a relação entre o exame e as condições sociais e 
econômicas das mulheres são bastantes significativas, não havendo comunicação entre os serviços e as usuárias e a percepção feminina em relação ao exame é deficiente.

Segundo Franco et al. (2019), além de maior cobertura de exames preventivos, deve-se agir para dar soluções dos exames alterados, pois quanto maior o número de exames realizados, maior a probabilidade de resultados alterados.

Dalmolin et al. (2016) observaram divergências no seu estudo entre resultados de exames e intervenções preconizadas na Diretriz Brasileira de Rastreamento. Grande parte dos exames com resultados "células escamosas atípicas indeterminadas provavelmente não neoplásicas" e "lesão intraepitelial de baixo grau" são encaminhados para outros níveis de atenção quando a recomendação é para que o tratamento seja realizado no nível local (Brasil 2016). Por outro lado, lesões que devem ser encaminhadas como "Células escamosas atípicas de significado indeterminado, quando não se pode excluir lesão intraepitelial de alto grau", "Células glandulares atípicas de significado indeterminado, possivelmente não neoplásico ou/e quando não se pode excluir lesão intraepitelial de alto grau", "Células atípicas de origem indefinida", são tratadas a nível local.

\section{Considerações finais}

O câncer do colo do útero se detectado e tratado precocemente pode atingir um índice de $100 \%$ de cura e ainda reduzir consideravelmente suas estatísticas quanto a mortalidade, por meio de ações que incluem o rastreamento eficiente. Para que o rastreamento seja uma medida de prevenção e controle eficiente, a cobertura precisa atingir um nível satisfatório. Considerando que o método utilizado no Brasil depende da busca das mulheres pelos serviços de saúde, o primeiro ponto a ser alterado seria no sentido de adoção de método de base populacional, o que garantiria a cobertura suficiente na realização do exame preventivo.

Nas mulheres que apresentam alterações no resultado do exame, o seguimento oportuno é fundamental. E para aprimorar esse serviço, a problemática do acesso às consultas precisa ser enfrentada, uma vez que, nesse caso, é de extrema importância que a consulta de retorno seja agilizada para evitar o desenvolvimento do câncer de colo do útero. As ações em saúde precisam ser implementadas de maneira oportuna, de forma a garantir uma completa recuperação e uma vida saudável à mulher, no sentido de diminuir as problemáticas e assistir as mulheres em sua integralidade.

A perda de seguimento ocorre devido a fatores como a má interpretação de exames, resultando em repetição do exame preventivo - o que aumenta os custos com a realização de exames desnecessários - e atrasa a realização de colposcopia e início do tratamento. Seguimentos ignorados de exames alterados, deficiência de orientação e encaminhamento destas mulheres evidenciam a fragmentação da atenção à saúde. A constituição de redes de atenção à saúde com funções pré-definidas dos três níveis de gestão governamental do SUS e pautadas pelas linhas de cuidado garantiriam um aprimoramento do cuidado no sentido da integralidade.

Destaca-se um importante aliado a este serviço, o preenchimento adequado das informações nos prontuários, uma vez que auxilia na busca ativa oportuna às mulheres que realizaram o exame para comparecerem à consulta de retorno.

$\mathrm{Na}$ organização do serviço, também podem ser desenvolvidos estudos diagnósticos que, por meio de um sistema de rastreamento, apontem os motivos para a não adesão das mulheres, identifiquem a disponibilidade do serviço à comunidade, obtenham controle de qualidade desses serviços, avaliem protocolos utilizados e o método de busca ativa das pacientes e garantam tratamento adequado às mulheres com alterações em seus exames. de saúde perante a comunidade.

Esta pesquisa levanta uma reflexão acerca da importância do alinhamento entre a realização dos exames citopatológicos e o acesso à consulta de retorno. Mostra a importância de seguir protocolos pré-estabelecidos, como os disponibilizados pelo Ministério da Saúde para aumentar a capacidade resolutiva dos casos encontrados. 


\section{Agradecimentos}

Agradecemos ao Curso de Especialização em Gestão de Redes de Atenção à Saúde da Escola Nacional de Saúde Pública da Fundação Oswaldo Cruz (ENSP-Fiocruz).

\section{Referências}

Araújo E.S., Barbosa F.M., Ázara C.Z.S., Ferreira T.X.A.M., Tavares S.B.N.M. \& Amaral R.G. (2014) Avaliação do seguimento de mulheres com exames citopatológicos alterados de acordo com as condutas preconizadas pelo Ministério da Saúde em Goiânia, Goiás. Revista Brasileira de Cancerologia, 60(1): 7-13.

Brasil (2010) Ministério da Saúde. Instituto Nacional do Câncer. Plano de ação para redução da incidência e mortalidade por câncer do colo do útero. Rio de Janeiro: INCA. 40 p.

Brasil (2011) Tribunal de Contas da União. Política Nacional de Atenção Oncológica. Brasília: TCU. 132 p.

Brasil (2013) Ministério da Saúde. Portaria no 3.394 de 30 de dezembro de 2013. Disponível em: https://bvsms.saude.gov.br/bvs/saudelegis/gm/2013/prt3394_30_12_2013.html (acessado em 01/07/2021).

Brasil (2016) Ministério da Saúde. Instituto Nacional do Câncer. Diretrizes brasileiras para o rastreamento do câncer do colo do útero. $2^{\circ}$ edição. Rio de Janeiro: INCA. 118 p.

Brasil (2021a) Ministério da Saúde. Instituto Nacional do Câncer. Câncer do colo do útero Fatores de Risco. Disponível em: https://www.inca.gov.br/controle-do-cancer-do-colo-doutero/fatores-de-risco (acessado em 30/05/2021).

Brasil (2021b) Ministério da Saúde. Instituto Nacional do Câncer. Câncer do colo do útero versão para profissionais. Disponível em: https://www.inca.gov.br/tipos-de-cancer/cancer-docolo-do-utero (acessado em 30/05/2021).

Brasil (2021c) Ministério da Saúde. Instituto Nacional do Câncer. Câncer do colo do útero Conceito e Magnitude. Disponível em: https://www.inca.gov.br/controle-do-cancer-do-colo-doutero/conceito-e-magnitude (acessado em 30/05/2021).

Brasil (2021d) Ministério da Saúde. Instituto Nacional do Câncer. Estatísticas de Câncer. Disponível em: https://www.inca.gov.br/numeros-de-cancer (acessado em 30/05/2021).

Caetano R., Vianna C.M.M., Thuler L.C.S. \& Girianelli V.R. (2006) Custo efetividade no diagnóstico precoce do câncer de colo uterino no Brasil. Physis: Revista de Saúde Coletiva, 16(1): 99-118. https://doi.org/10.1590/S0103-73312006000100007

Carvalho V.F., Kerber N.P.C., Wachholz V.A., Pohlmann F.C., Marques L.A. \& Francioni F.F. (2016) Acesso ao exame Papanicolau por usuárias do sistema único de saúde. Revista Rene, 17(2): 198-207.

Carvalho P.G., O'Dwer G. \& Rodrigues N.C.P. (2018) Trajetórias assistenciais de mulheres entre diagnóstico e início de tratamento do câncer do colo uterino. Saúde Debate, 42(118): 687-701.

Casarin M.R. \& Piccoli J.C.E.B. (2011) Educação em saúde para a prevenção do câncer de colo do útero em mulheres do município de Santo Ângelo/MS. Ciências \& saúde coletiva, 16(9): 3925-3932. https://doi.org/10.1590/S1413-81232011001000029

Cruz L.M.B. \& Loureiro R.P. (2008) A comunicação na abordagem preventiva do câncer do colo do útero: importância das influências histórico-culturais e da sexualidade feminina na adesão às campanhas. Saúde Sociais, 17(2): 120-131. https://doi.org/10.1590/S0104-12902008000200012

Dalmolin S.P., Dexheimer G.M. \& Delving L.K.O.B. (2016) Mulheres com exames citopatológicos alterados: Avaliação do seguimento de acordo com as condutas preconizadas pelo Ministério da Saúde. Revista Brasileira de Análise Clínicas, 48(3): 235-239.

Farias A.C.B. \& Barbieri A.R. (2016) Seguimento do câncer de colo do útero: Estudo da continuidade da assistência à paciente em uma região de saúde. Escola Ana Nery, 20(4): e20160096. https://doi.org/10.5935/1414-8145.20160096 
Febrasgo (2017) Rastreio, diagnóstico e tratamento do câncer de colo de útero. São Paulo: Federação Brasileira das Associações de Ginecologia e Obstetrícia (FEBRASGO). 64 p.

Franco J.L.V., Gondinho B.V.C., Bulgareli J.V., Cortellazzi K.L., Guerra L.M., Costa A.D., Cavalcante D.F.B., Ambrosano G.M.B. \& Pereira A.C. (2019) Seguimento de Resultados Alterados de Papanicolau nas Unidades Básicas de Saúde do Município de São Paulo. Revista Eletrônica Gestão \& Saúde, edição especial: 19-36. https://doi.org/10.26512/gs.v0i0.23317

Gil A.C. (2011) Como elaborar projetos de pesquisa. $5^{\circ}$ edição. São Paulo: Atlas. 188 p.

Köche J.C. (2011) Fundamentos de metodologia científica: teoria da ciência e iniciação a pesquisa. $29^{\circ}$ edição. Petrópolis: Vozes. 185 p.

Ribeiro M.G.M., Santos S.M.R. \& Teixeira M.T.B. (2011) Itinerário terapêutico de mulheres com câncer do colo do útero: uma abordagem focada na prevenção. Revista Brasileira de Cancerologia, 57(4): 83-91. https://doi.org/10.32635/2176-9745.RBC.2011v57n4.644

Risso B.B.S. \& Soares M.C. (2011) Avaliação dos registros do exame citopatológico em uma unidade básica de saúde em Rio Grande-RS. Revista de Enfermagem em Saúde, 1(1): 84-90. https://doi.org/10.15210/jonah.v1i1.3410

Santos J.G.C. \& Dias J.M.G. (2018) Vacinação pública contra o papilomavirus humano no Brasil. Revista Médica de Minas Gerais, 28: 2238-3182.

Silva D., Lopes E.L. \& Braga S.S. (2014) Pesquisa Quantitativa: elementos, paradigmas e definições. Revista de Gestão e Secretariado - GeSec, 5(1): 1-18. https://doi.org/10.7769/gesec.v5i1.297

Soares S.J. (2019) Pesquisa Científica: Uma abordagem sobre o Método Qualitativo. Revista Ciranda, 3(1): 168-180.

Tavares S.B.N., Amaral R.G., Manrique E.J.C., Souza N.L.A., Albuquerque Z.B.P. \& Zeferino L.C. (2007) Controle da qualidade em citopatologia cervical: revisão de literatura. Revista Brasileira de Cancerologia, 53(3): 355-364.

Uchimura N.S., Nakano K., Nakano L.C.G. \& Uchimura T.T. (2009) Qualidade e desempenho das colpocitologias na prevenção de câncer de colo uterino. Revista da Associação de Medicina Brasileira, 55(5): 569-574. https://doi.org/10.1590/S0104-42302009000500021

Vale D.B.A.P., Morais S.S., Pimenta A.L. \& Zeferino L.C. (2010) Avaliação do rastreamento do câncer do colo do útero na Estratégia Saúde da Família no Município de Amparo, São Paulo, Brasil. Caderno de Saúde Pública, 26(2): 383-390. https://doi.org/10.1590/S0102-311X2010000200017

Vieira S. \& Hossene W. (2007) Metodologia científica para a área da saúde. Rio de Janeiro: Elsevier. 175 p. 